\title{
Performance Analysis of a Downlink MAC Protocol with Power-Saving Support
}

\author{
Apostolis K. Salkintzis, Member, IEEE, and Christodoulos Chamzas, Senior Member, IEEE
}

\begin{abstract}
This paper presents the theoretical analysis of a downlink MAC protocol, which features power-saving characteristics and has been previously proposed [3], [7] for implementation in mobile data networks. The main objective of the protocol is to conserve valuable battery energy at the portable terminals of a wireless network. To accomplish this, the terminals employ discontinuous reception, i.e., they periodically power on and off their receive units. The key feature of the protocol, which makes it different from the other power-saving alternatives, is that it does not require system synchronization so the terminals are free to turn on and off their receivers based on their own necessities. The paper discusses the main properties of this protocol and develops a theoretical framework to approximate its performance. Theoretical results for the mean downlink packet delay as well as for the mean downlink power consumption are derived, and these results are compared against simulation outcomes in order to estimate their validity. The main conclusions are that the considered power-saving protocol features great potentials and can be easily implemented and that the developed theoretical model provides an acceptable theoretical tool for its analysis.
\end{abstract}

Index Terms-Energy consumption, energy saving, mobile data networks, power management, power saving, protocol analysis, wireless communications.

\section{INTRODUCTION}

$\mathbf{I}$ $\mathrm{N}$ AN ERA of emerging PCS technologies and sophisticated mobile services, the power-saving issue is becoming more and more important. It progressively becomes evident that some means for power saving should be provided for the wireless/portable terminals to be capable to support extensive service utilization. This is justified by the fact that the most remarkable wireless systems today, such as MOBITEX, CDPD, TETRA, IEEE 802.11, HIPERLAN, paging networks, etc., employ some kind of power-saving protocol.

The usual approach to reduce the power consumption of the portable terminals is to let them employ discontinuous reception, i.e., to let them periodically power off their receive units (go to "sleep" mode) instead of continuously listening to the radio channel. This discontinuous reception can be proved very efficient in terms of power saving because as Stemm et al. showed for typical usage scenarios, by far the most power (about 90\%) is drawn by listening to the radio

Manuscript received November 24, 1997; revised January 4, 1999. This work was supported by the General Secretariat of Research and Technology of Greece under the project PENED 407.

A. K. Salkintzis is with the Department of Electrical and Computer Engineering, University of British Columbia, Vancouver, B.C., V6T 1Z4, Canada (e-mail: salki@ece.ubc.ca).

C. Chamzas is with the Department of Electrical and Computer Engineering, Democritus University of Thrace, Xanthi, Greece.

Publisher Item Identifier S 0018-9545(00)02571-8. channel [1], [2]. Packet transmission and reception contribute only a few percent to the energy balance.

There are some fundamental problems to be resolved before the wireless terminals can benefit from the discontinuous reception. First, it should be specified how a wireless terminal will receive data since it is not always listening to the radio channel and how it will send data to other terminals in sleep mode [2].

Generally, these problems found different solutions in different systems [2], [7], [10]-[13], but there are also some noticeable characteristics that apply to all of them. For example, data for terminals in power-saving mode is temporary buffered at the transmitter and is forwarded to them only after they get ready to receive data. Also, terminals in power-saving mode periodically "wake up" and listen to the radio channel in order to identify whether there is pending data for them or not.

Another common characteristic of the power-saving protocols employed today is the synchronization of the terminals' wake-up instants. Terminals are synchronized to wake up at the same time in which an announcement is transmitted that identifies the terminals with pending traffic. In cases where there is a central authority in the system, this authority provides the reference timing for synchronization and it also specifies the time periods where the terminals should wake up for downlink channel monitoring. During these wake-up periods (also referred to as "alert windows"), the central authority transmits special management frames [traffic announcement frames (TAF's)] that identify the terminals with buffered traffic. In ad hoc networks, such as IEEE 802.11 [6] and HIPERLAN [16], time synchronization is performed in a distributed fashion. It is to be noted, however, that in this paper we mainly focus on infrastructure networks where a central authority (e.g., a base station) always exists. This is the case for mobile data networks and can also be the case for wireless LAN's (although these LAN's can operate in ad hoc configuration as well).

Some systems, such as MOBITEX [4], force the wireless terminals to wake up at every alert window, and therefore they guarantee that TAF's sent by the central authority are received by all terminals. In such cases, it is implicitly known that after the transmission of a TAF all the announced terminals will be ready for data reception, and hence their buffered traffic can be transmitted directly. Other systems, such as CDPD [5] and IEEE 802.11 [6], do not force the wireless terminals to wake up at every alert window, and therefore it cannot be identified which terminals are ready for reception after a TAF. In such cases, the wireless terminals that actually get ready for reception provide an explicit notification to the central authority by transmitting a special frame [a receiver ready notification (RRN)]. On the one hand, these transmissions consume extra power, but on the other 
hand, the terminals can remain for longer periods in sleep mode whenever their communication needs are reduced.

As opposed to the above synchronous power-saving approach, where the terminals may (or must) wake up only when an alert window is scheduled, a different class of power-saving protocols have been proposed by the authors [3], [7]-[9], where the wireless terminals wake up whenever they want to and therefore asynchronously to each other. As indicated in [3] and in more details in [7], these protocols (referred to as asynchronous power-saving protocols) exhibit some notable advantages over their synchronous counterparts. First, they lack any synchronization, which means that no communication means are necessary to distribute a timing reference. Second, the terminals are free to wake up whenever they want to, and they do not have to keep track of the instants where alert windows occur. (It should be noted that when the period between successive alert windows changes, ${ }^{1}$ a wireless terminal in a synchronous system can keep track of the alert windows only if it wakes up at every alert window. Obviously, this causes limitations to its power management.) Third, since the terminals wake up asynchronously, the transmissions of RRN are less likely to collide as compared to the synchronous wake-up case (where many terminals may try to transmit a RRN at the same time). For this reason, RRN retransmissions may be considerably reduced in an asynchronous protocol and an extra power-saving gain could be expected. For more details on the advantages and the disadvantages of asynchronous power-saving protocols the reader is referred to [3] and [7].

The purpose of this paper is to theoretically study some performance measures of an asynchronous power-saving protocol, namely the in-band power-saving (IPS) protocol. This protocol has been introduced in [3] and has been studied with the aid of computer simulation. Various figures of merit of this protocol have been evaluated for various scenarios (e.g., for exhaustive, first-in-first-out, and priority service disciplines), and additionally it has been shown how the downlink performance is degraded due to the power saving. In this paper, however, we restrict our attention to the theoretical treatment of the IPS protocol. In particular, we develop closed theoretical formulas for some important performance measures of this protocol, and we compare the derived theoretical results against our simulation results. By this way we also evaluate the accuracy and the validity of the formulated theoretical model.

The rest of the paper is organized as follows. In Section II, the general operational description of the IPS protocol takes place and its primary procedures and elements are outlined. In Section III, we develop the theoretical analysis of the protocol based on a number of assumptions. These assumptions are explained and briefly discussed. Also, theoretical results for both the mean downlink packet delay and mean downlink power consumption are graphically presented and compared to the corresponding simulation results. Furthermore, a case where the discontinuous operation of the terminals is adapted to the downlink

\footnotetext{
${ }^{1}$ Within an alert window the central authority transmits information identifying the instant where the next alert window will occur. It is possible for this information to change from time to time in accordance to the volume of the buffered traffic.
}

traffic conditions is analyzed through computer simulation. Finally, in Section IV we summarize our conclusions.

\section{DESCRIPTION OF THE IPS PROTOCOL}

The main objective of the IPS protocol is to allow mobile terminals to implement their own independent and dynamic power management algorithm and obtain an optimum balance between packet delay and battery consumption. In this way, a terminal will be able to select a proper duty cycle ${ }^{2}$ for its receiver. For example, at healthy battery periods, a long duty cycle (or even a continuous reception) could be selected to maximize downlink throughput, whereas at low battery conditions a short duty cycle could be selected to offer increased service utilization even with degraded performance.

A detailed and comprehensive description of the IPS protocol can be found in [3] and [7]. However, in order to maintain the clarity and the legibility of the paper a concise verbal description is also provided below.

We consider a centralized data communication environment where one base station serves a number of wireless-portable terminals as illustrated in Fig. 1. Two frequency-multiplexed channels support the communication between the base station and the terminal fleet: the uplink, for data transfer from the terminals to the base station, and the downlink, for data transfer in the opposite direction. Portable terminals operate their receivers intermittently, that is, they periodically withdraw the power supply from them. As already mentioned, they are free to operate theirs receivers whenever they like to; they are not synchronized to a common reference clock and therefore they do not necessarily wake up at the same time. In such case, the IPS protocol is implemented to provide an answer to the question "how can the buffered traffic in the base station be delivered to the terminals?"

Packets arriving at the base station and destined to the portable terminals are temporary buffered instead of being immediately forwarded. Whenever there is buffered traffic the base station transmits page messages that identify the downlink traffic demand; in other words, they identify the terminals that the buffered traffic should be forwarded to. The paging performed by the base station is continuous. One page message succeeds the other until at least one terminal declares that it is ready for reception. In this case, paging is suspended and the buffered traffic for the ready terminal(s) is transmitted. After data transmission, paging resumes provided that there is residual buffered traffic. ${ }^{3}$

A terminal willing to check if there is pending traffic for it powers up its receiver and monitors the downlink channel for some short time. Should it receive a page message including its own address, it remains in receive mode and it also notifies the base station that it is ready for reception. This is done by the transmission of a short message called Receiver-Ready Notification (RRN). In this way, the base station learns its reception capability and proceeds to data transmission. Of course, when

\footnotetext{
${ }^{2}$ The duty cycle is defined as the wake-up period over the sum of wake-up and sleep periods.

${ }^{3}$ Of course, in a real system the base station will not page a given terminal forever. After a maximum paging period, it will assume that the terminal is shut down and it will stop paging it.
} 


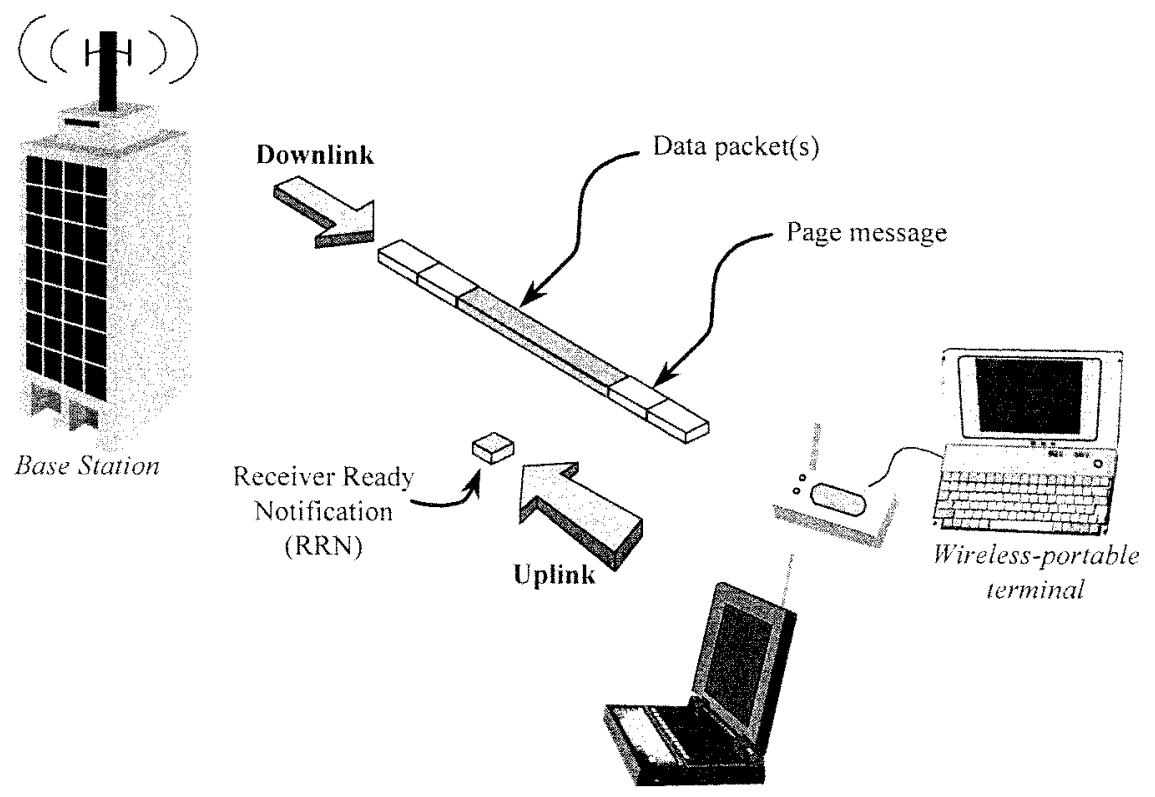

Fig. 1. The considered mobile data network.

the terminal receives no information indicating the presence of pending traffic it switches its receiver off.

\section{Performance AnAlysis}

In this section, we study the performance analysis of the IPS. The theoretical analysis is based on some simplifying assumptions that one should consider for making the analysis feasible. We mainly focus on the mean packet delay and the downlink power consumption of the portable terminals.

\section{A. Mean Packet Delay}

The mean packet delay $\bar{T}$ is defined as the average time period between the arrival of a packet to the base station and the delivery of this packet to the addressed terminal. In the case of the considered protocol, this mean packet delay can be written as

$$
\bar{T}=\bar{A}+\bar{D}
$$

where $\bar{A}$ is the mean paging delay and $\bar{D}$ is the mean service delay. The paging delay $\tilde{A}^{4}$ for a packet addressed to terminal $m$ is defined as the time period from the packet arrival to the base station until the terminal $m$ transmits an RRN message, i.e., until the terminal $m$ gets ready for reception. On the other hand, the service delay $\tilde{D}$ is defined as the period from the time that the base station receives the RRN message until the packet is finally received by the addressed terminal. Obviously, this period stands for a queueing delay. We note that $\tilde{A}, \tilde{D}$ are continuous random variables with possible values in the range $[0 \cdots \infty)$.

Below we calculate closed formulas for the average values $\bar{A}$ and $\bar{D}$ of these random variables by making some simplifying assumptions. It is important to note that these assumptions may not be the most practical ones, however, they are sufficient for building up a tractable and valuable theoretical model which can be compared to classical queueing models like M/G/1 or

\footnotetext{
${ }^{4} \tilde{A}$ stands for the random variable and $\bar{A}$ stands for its average value.
}

M/M/1 [14], [15]. By such comparisons, we can evaluate the effects of the asynchronous discontinuous reception to the system performance, e.g., we can estimate the downlink throughput degradation (compared to the case of continuous reception), and the power consumption gain. Our assumptions are summarized below.

1) Packet arrivals at the base station follow a Poisson distribution with total average arrival rate $\lambda$.

2) Packet transmission time is exponentially distributed with an average value $1 / \mu(\mu>\lambda)$.

3) The network is composed of $M$ portable terminals which are independent and statistically identical to each other. Therefore, the average packet arrival rate per terminal is $\lambda_{s}=\lambda / M$.

4) The buffering capabilities of the base station are considered infinite.

5) The base station implements an exhaustive service discipline for packet transmission, that is, whenever it services a terminal it transmits all its pending packets.

6) RRN transmissions do not occur simultaneously.

7) We assume that an arbitrary page message is received by a terminal with probability $p$ (which is considered the same for every terminal). This probability $p$ will be referred to as page message reception probability, and it virtually models the discontinuous reception of the terminals. In particular, probability $p$ is equal to the receiver's duty cycle and is considered to be constant and independent to the traffic volume. However, in a later section we address a more practical case where the probability $p$ changes with the traffic volume.

8) The transmission duration of a page message is constant (that is independent to the number of terminals with buffered traffic) and equal to $s$.

9) Without introducing a large error, we assume that the probability a packet addressed to a terminal $m$ to arrive during the service period of terminal $m$ is zero. 


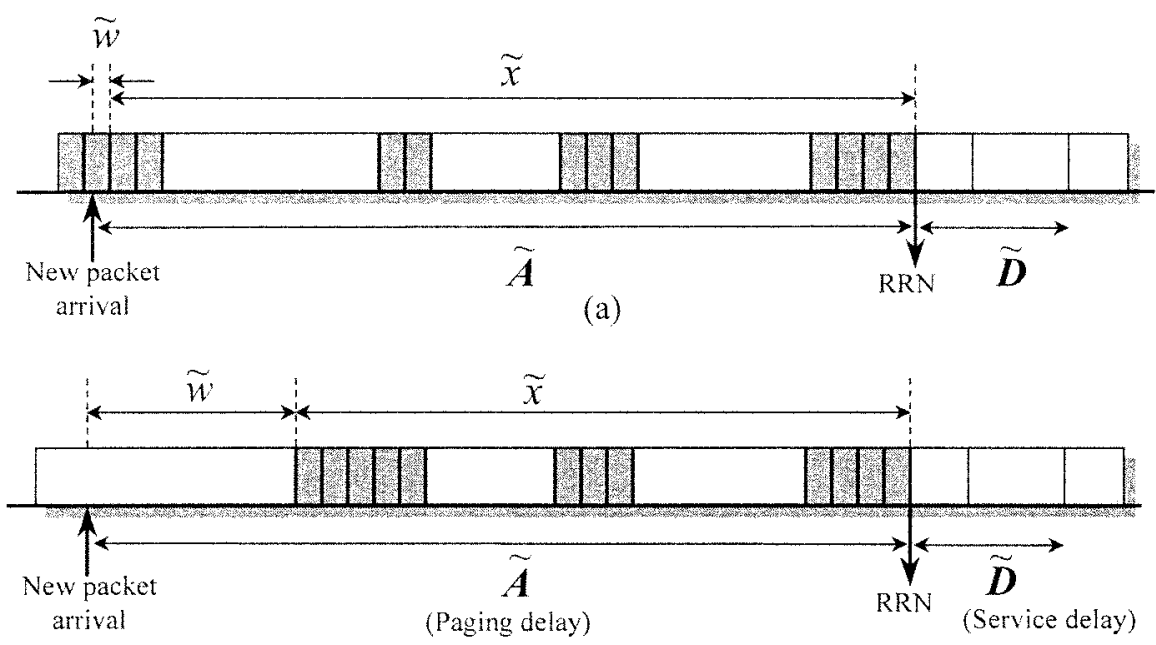

(b)

Fig. 2. Definition of the paging delay and the service delay.

10) Finally, we assume that RRN messages do not get destroyed nor delayed during their transmission on the uplink channel (of course, this will lead us to a best case performance).

As indicated in Fig. 2, the paging delay $\tilde{A}$ can be decomposed into two independent random variables $\tilde{w}$ and $\tilde{x}$. The time interval $\tilde{w}$ is the period from a packet arrival until the beginning of the next page message, while the time interval $\tilde{x}$ represents the period from the beginning of the next page message until the base station receives an RRN from the terminal that the arrived packet is addressed to.

Generally, the time interval $\tilde{w}$ depends on the downlink channel state at the arrival instant of the packet. In Fig. 2 two cases are shown: in (a) the packet arrives while there are page messages transmitted on the downlink channel, whereas in (b) the packet arrives while there are data packets transmitted on the downlink channel. Of course, a packet arrival may as well occur while the downlink channel is idle and in this case the time interval $\tilde{w}$ is zero. ${ }^{5}$

We can consider the downlink channel as a stochastic process taking three individual states: $D$ (data transmission), $B$ (paging transmission), and $I$ (idle). The probabilities of the states $D, B$, and $I$ are defined as $P_{D}, P_{B}$, and $P_{I}$, respectively.

Now we can write the average value of time interval $\tilde{w}$ as

$$
E[\tilde{w}]=\bar{D}_{R} P_{D}+\frac{s}{2} P_{B}+0 P_{I}=\bar{D}_{R} P_{D}+\frac{s}{2} P_{B}
$$

where $\bar{D}_{R}$ is the average residual service period [the average value of $\tilde{w}$ in Fig. 2(b)] and $s$ is the transmission duration of a page message. Based on the principles of renewal theory, if the average duration of the service period is $\bar{D}$ and if the variance of the service period is $\sigma^{2}$, then the average residual service period is ([15], p. 173)

$$
\bar{D}_{R}=\frac{\bar{D}}{2}+\frac{\sigma^{2}}{2 \bar{D}} .
$$

${ }^{5}$ Interval $\tilde{w}$ may as well be zero when a packet for terminal $m$ arrives during the service period of terminal $m$. However, according to assumption 9), the probability of this event is considered zero for simplicity.
Assuming that $\alpha$ packets are transmitted, the service period has a gamma distribution with variance $\sigma^{2}=\alpha / \mu^{2}$, therefore, $\bar{D}_{R}$ can be written as

$$
\bar{D}_{R}=\sum_{\alpha=1}^{\infty}\left(\frac{\bar{D}}{2}+\frac{\alpha}{2 \mu^{2} \bar{D}}\right) P(\alpha)
$$

where $P(\alpha)$ is the probability $\alpha$ packets to be transmitted in a service period. We approximate $P(\alpha)$ with a Poisson distribution with parameter $\lambda_{s} \bar{A}$; as justified by the simulation results the error of this approximation is less than $0.1 \%$ for $\lambda / \mu<0.9$. Therefore, (4) can be written as

$$
\begin{aligned}
\bar{D}_{R} & \cong \sum_{\alpha=1}^{\infty}\left(\frac{\bar{D}}{2}+\frac{\alpha}{2 \mu^{2} \bar{D}}\right) \frac{\left(\lambda_{s} \bar{A}\right)^{\alpha}}{\alpha !} e^{-\lambda_{s} \bar{A}} \\
& =\frac{\bar{D}}{2}\left(1-e^{-\lambda_{s} \bar{A}}\right)+\frac{\lambda_{s} \bar{A}}{2 \mu^{2} \bar{D}} .
\end{aligned}
$$

We now proceed to the calculation of the mean service period $\bar{D}$ and the probabilities $P_{D}$ and $P_{B}$.

As already defined, the paging delay $\tilde{A}$ is the time period from the arrival instant of a packet for some terminal $m$ until that terminal transmits an RRN message. In Fig. 3, we illustrate the arrival instants of three typical packets and their corresponding paging delays $\tilde{A}_{1}, \tilde{A}_{2}, \tilde{A}_{3}$. An important observation is that the random variables $\tilde{A}_{1}, \tilde{A}_{2}, \tilde{A}_{3}, \cdots$ have identical distributions; in other words, the paging delay does not depend on which packet we choose to measure it. This effect is due to the assumption 7) and specifically due to the fact that the probability a terminal to receive a page message does not depend on the time when the paging procedure started (memoryless property). For this reason, we can write that $\bar{A}=\bar{A}_{1}=\bar{A}_{2}=\bar{A}_{3}=\cdots$.

Generally, when a terminal transmits an RRN message, there will be $\tilde{N}=1,2,3, \cdots$ pending packets (see Fig. 3 ) for it at the base station. According to the above observation the average value of $\tilde{N}$ will be given by the following:

$$
\bar{N}=\lambda_{s} \bar{A}_{1}+1=\lambda_{s} \bar{A}+1
$$




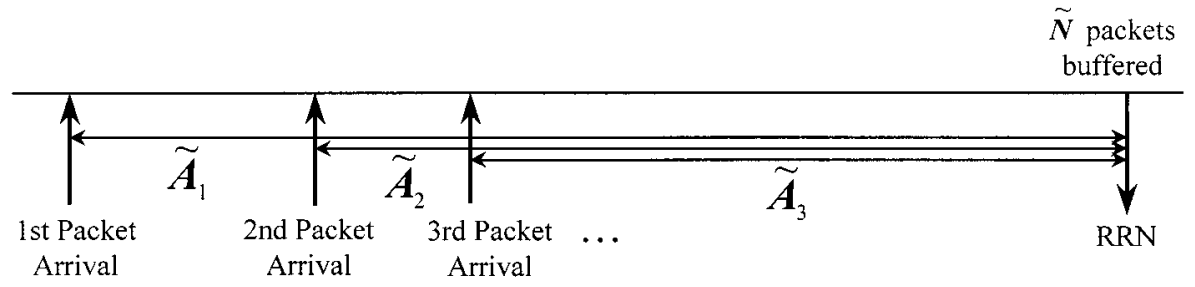

Fig. 3. Paging delay corresponding to three sample packets.

where $\lambda_{s}$ is the average traffic rate per terminal. From (6) and provided that the average packet transmission time is $1 / \mu$, we calculate the average service period

$$
\bar{D}=\frac{\lambda_{s} \bar{A}+1}{\mu} .
$$

Regarding probability $P_{D}$ one can readily show that is equal to the downlink channel utilization factor $\rho \equiv \lambda / \mu$. If we have $\lambda x$ new packet arrivals in a time period $x$ and if within every $D$ state there are $\lambda_{s} \bar{A}+1$ packets transmitted on the average, then the relative occurrence frequency of $D$ state is (we assume equilibrium where the average packet arrivals and departures within period $x$ are the same)

$$
\begin{aligned}
R_{D} & =\lim _{x \rightarrow \infty} \frac{\text { number of } D \text { states in period } x}{x} \\
& =\frac{\lambda}{\lambda_{s} \bar{A}+1} .
\end{aligned}
$$

It is easy to verify that

$$
P_{D}=R_{D} \bar{D}=\rho .
$$

Writing (9) for the $B$ state we have

$$
P_{B}=R_{B} \bar{B}=R_{D} \bar{B}=\frac{\lambda}{\lambda_{s} \bar{A}+1} \bar{B} .
$$

We could have accurately calculated $\bar{B}$ if we knew the distribution of the random variable $\tilde{v}$, which expresses the number of terminals with pending traffic at the moment where a new $B$ state initiates. However, since this distribution is not easily obtained we resort to the following approximate method. We assume that $\bar{B}$ can be expressed by a second-order polynomial

$$
\bar{B}=y_{1} \rho^{2}+y_{2} \rho+y_{3}
$$

where $y_{1}, y_{2}, y_{3}$ are constant coefficients and independent of $\lambda$, $\rho$. We select these coefficients as follows.

When $\rho \rightarrow 0$, the base station will page only one terminal every time a paging procedure takes places, and therefore a paging sequence will last until this terminal replies with an RRN. The probability the terminal to transmit an RRN after $k$ pages is $p(1-p)^{k-1}$, where $p$ is the reception probability defined in assumption 7). So

$$
\bar{B}(\rho=0)=\sum_{k=1}^{\infty}(k s) p(1-p)^{k-1}=\frac{s}{p} .
$$

Likewise, when $\rho \rightarrow 1$, the base station will page all terminals at every paging procedure and therefore

$$
\begin{aligned}
\bar{B}(\rho=1) & =\sum_{k=1}^{\infty}(k s)\left[1-(1-p)^{M}\right](1-p)^{M(k-1)} \\
& =\frac{s}{1-(1-p)^{M}} .
\end{aligned}
$$

From (11) to (13), we have

$$
y_{3}=\frac{s}{p} \quad \text { and } \quad y_{1}+y_{2}=\frac{s}{1-(1-p)^{M}}-\frac{s}{p}<0 .
$$

From the simulation results, we observe that (11) will have to decline as $\rho$ moves from zero to one and that its second derivative $\left(2 y_{1}\right)$ will have to be negative. Furthermore, its first derivative will have to be zero at a value $\rho<0$, and therefore $-y_{2} / 2 y_{1}<0$. From these considerations, we conclude that $y_{1}$, $y_{2}$ will have to be negative and can be written as

$$
y_{1}=\frac{-s}{p}-x \quad \text { and } \quad y_{2}=\frac{s}{1-(1-p)^{M}}-x
$$

where $x$ is a real number. Although any value of $x$ in the space $\left(s / 1-(1-p)^{M}, s / p\right)$ will make $y_{1}, y_{2}$ negative, $x$ will have to depend on the ratio $s / p$ in order to have a good approximation. Considering all the above, the following values for the coefficients $y_{1}, y_{2}, y_{3}$ were chosen:

$$
\begin{aligned}
& y_{1}=\frac{-s}{p}+\frac{s}{p}\left[\frac{s / p+s / 1-(1-p)^{M}}{2}\right] \\
& y_{2}=\frac{s}{1-(1-p)^{M}}-\frac{s}{p}\left[\frac{s / p+s / 1-(1-p)^{M}}{2}\right] \\
& y_{3}=\frac{s}{p} .
\end{aligned}
$$

Comparing the values of (11) [with the coefficients of (15a)-(15c)] against our simulation results, it can be claimed that the above approximation of $\bar{B}$ is quite good and specifically when the ratio $s / p$ is large.

Finally, from (2), (5), (7), (9), and (10), we derive the average duration of period $\tilde{w}$ as

$$
\begin{aligned}
E[\tilde{w}]= & {\left[\frac{\bar{D}}{2}\left(1-e^{-\lambda_{s} \bar{A}}\right)+\frac{\lambda_{s} \bar{A}}{2 \mu^{2} \bar{D}}\right] \rho } \\
& +\frac{\lambda s}{2\left(\lambda_{s} \bar{A}+1\right)}\left[y_{1} \rho^{2}+y_{2} \rho+\frac{s}{p}\right]
\end{aligned}
$$

where $y_{1}, y_{2}$ are given by (15a) and (15b), respectively.

In the following, we calculate the average duration of time period $\tilde{x}$ (see Fig. 4). Generally, this period is composed of $\tilde{k}$ 


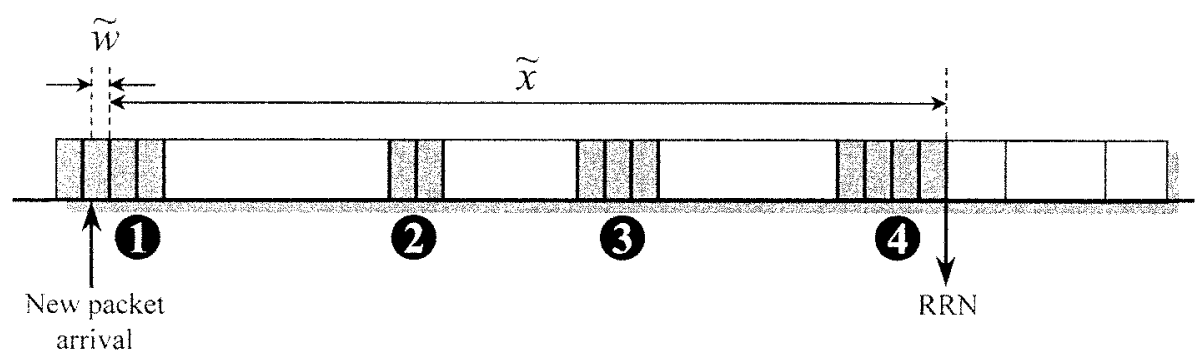

Fig. 4. Time delay $\tilde{x}$ is composed of $\tilde{k}$ paging sequences and $(\tilde{k}-1)$ service periods. In this example, $\tilde{k}=4$.

paging sequences ( $B$ states) and $(\tilde{k}-1)$ service periods $(D$ states), where $\tilde{k}=1,2,3, \cdots$ is a discrete random variable. It is evident that the $(\tilde{k}-1) D$ states are independent and uniformly distributed. The same holds true for the $\tilde{k} B$ states, even when the packet arrival occurs during a $B$ state (according to assumption 7) the $B$ state has a geometrical distribution so its duration is memoryless). Therefore, the average value of $\tilde{x}$ can be written as

$$
E[\tilde{x}]=\bar{k} \bar{B}+(\bar{k}-1) \bar{D}
$$

where the average values $\bar{B}, \bar{D}$ are given by (11) and (7), respectively. The quantity $\bar{k}-1$ expresses the average number of terminals that get serviced before the considered terminal transmits its RRN message. If $P_{N}$ is the probability a paging sequence to be composed of $N$ pages, then the probability $\tilde{k}$ to equal $n=1,2,3, \cdots$ is

$$
\operatorname{Pr}[\tilde{k}=n]=(1-X) X^{n-1}
$$

where

$$
X \equiv \sum_{N=1}^{\infty}(1-p)^{N} P_{N}
$$

Quantity $X$ stands for the probability a terminal to not send an RRN message at the end of a paging sequence, therefore, the average value of $\tilde{k}$ is

$$
\bar{k}=\sum_{n=1}^{\infty} n X^{n-1}(1-X)=\frac{1}{1-X} .
$$

By setting $z=1-p$ in (19), we have

$$
X=\sum_{N=1}^{\infty} z^{N} P_{N}=\sum_{N=0}^{\infty} z^{N} P_{N}=E\left[z^{N}\right] \equiv G(z)
$$

where $G(z)$ is the probability generating function of the random variable $\tilde{N}$, which represents the number of page messages that compose a page sequence. To avoid the complex calculation of the distribution $P_{N}$, we make the following approximation:

$$
G(z) \cong G(z=1)+\left.(z-1) \frac{d G(z)}{d z}\right|_{z=1} .
$$

Provided that $z-1=(1-p)-1=-p$, this approximation is getting more and more accurate as the reception probability $p$ is getting smaller and smaller. From (21), we derive that $G(z=$
1) $=1$, and from (11) and the properties of the probability generating functions [15], we obtain that

$$
\left.\frac{d G(z)}{d z}\right|_{z=1}=E[\tilde{N}]=\frac{y_{1}}{s} \rho^{2}+\frac{y_{2}}{s} \rho+\frac{1}{p} .
$$

Using (20)-(23), we end up with

$$
\bar{k}=\frac{s}{s+p\left(y_{1} \rho^{2}+y_{2} \rho\right)} .
$$

Finally, (17) with the aid of (7), (11), and (24) becomes

$$
\begin{aligned}
E[\tilde{x}]= & \frac{s}{s+p\left(y_{1} \rho^{2}+y_{2} \rho\right)}\left[y_{1} \rho^{2}+y_{2} \rho+\frac{s}{p}+\frac{\lambda_{s} \bar{A}+1}{\mu}\right] \\
& -\frac{\lambda_{s} \bar{A}+1}{\mu} .
\end{aligned}
$$

Now, the average paging delay $\bar{A}$ can be found by adding (16) and (25), and afterwards the average packet delay $\bar{T}$ can be readily evaluated by using (1) and (7).

In order to evaluate the results of the above analysis, we depict in Fig. 5(a)-(d) the theoretical and simulation curves of the mean downlink packet delay versus the downlink channel utilization $\rho=\lambda / \mu$. The figures correspond to four different reception probabilities $p$ and to $M=20, s=0.1$ (our time unit is the average packet transmission period). For comparison, the average packet delay of continuous reception is also displayed.

As we observe, the theoretical curves furnish a good approximation of the average downlink packet delay specially when the ratio $s / p$ is large and the downlink channel utilization $\rho$ is not too high. Another observation (not perceptible from Fig. 5) is that the theoretical results are getting more and more accurate as the number of portable terminals is getting more and more small. This behavior is due to the simplifying assumptions and particularly due to the approximate calculation of the average value $\bar{k}$ and due to the assumption that there are no simultaneous RRN's.

The fact that the theoretical and simulation curves deflect from each other as the downlink traffic increases $(\rho \rightarrow 1)$ is caused primarily by two reasons. First, by the approximate evaluation of $P(\alpha)$ [see (5)] and second by the fact that (24) gives small values to the variable $\bar{k}$ when the downlink traffic is high. Therefore, theoretical results end up to smaller (more optimistic) mean packet delay at high traffic conditions. Comparatively to the continuous reception, we may note that the mean packet delay in IPS protocol with $p=0.05$ is typically degraded by a factor of about 3.4 over all the practical traffic conditions. However, the mean packet delay degradation remain below 1.5 as long as the reception probability $p$ is not smaller than 0.2 [3]. 


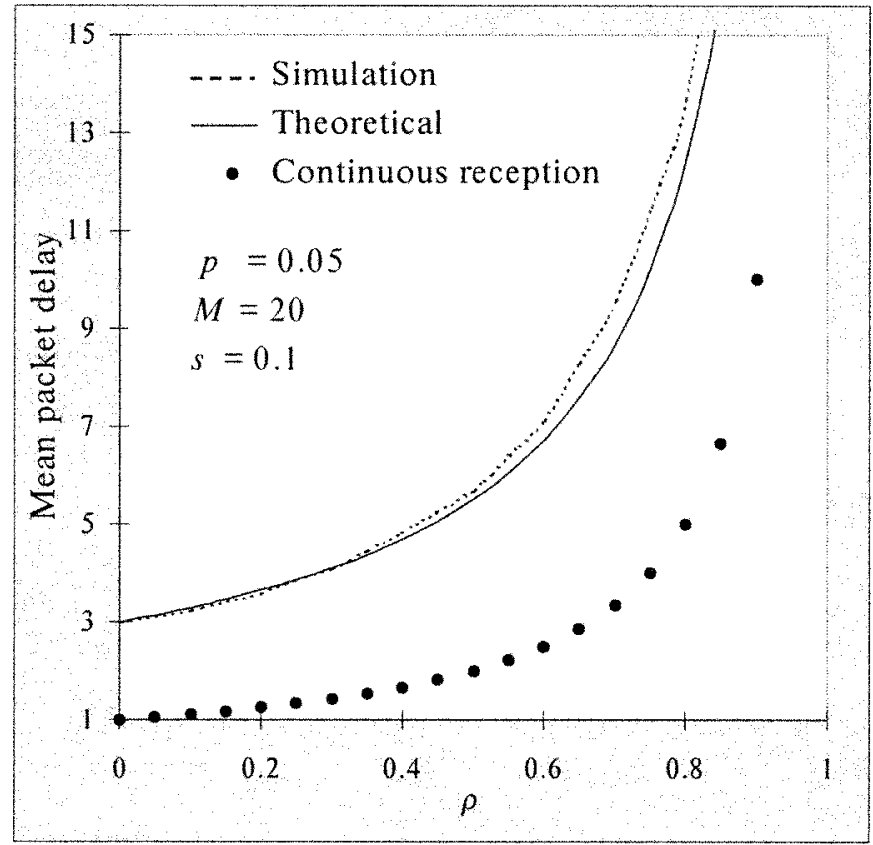

(a)

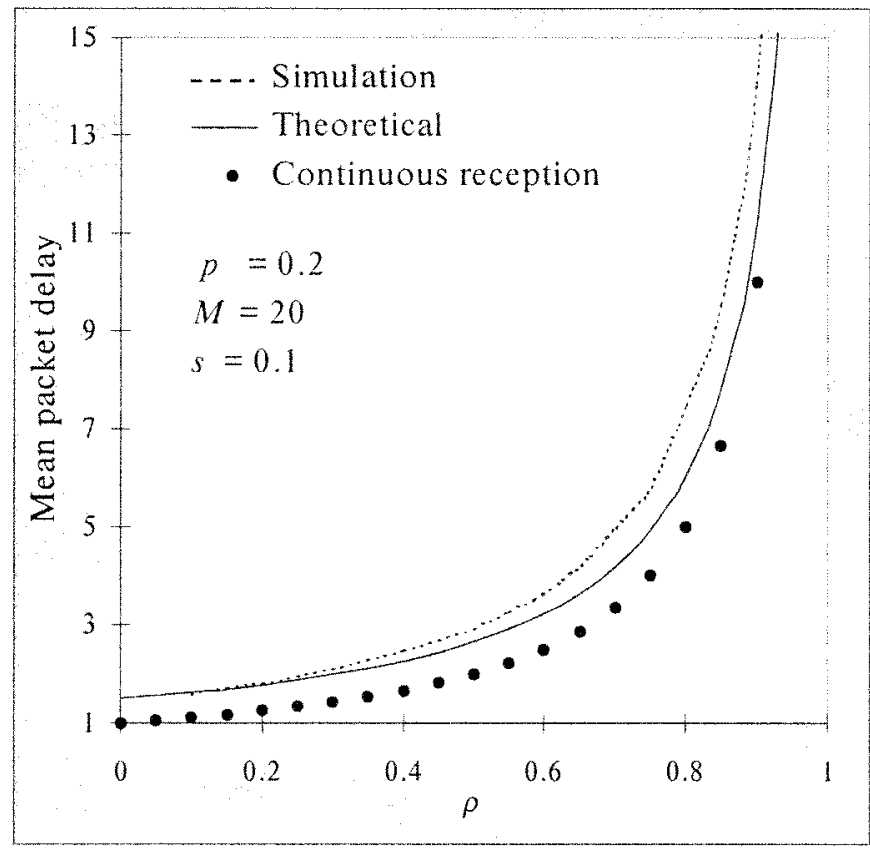

(c)

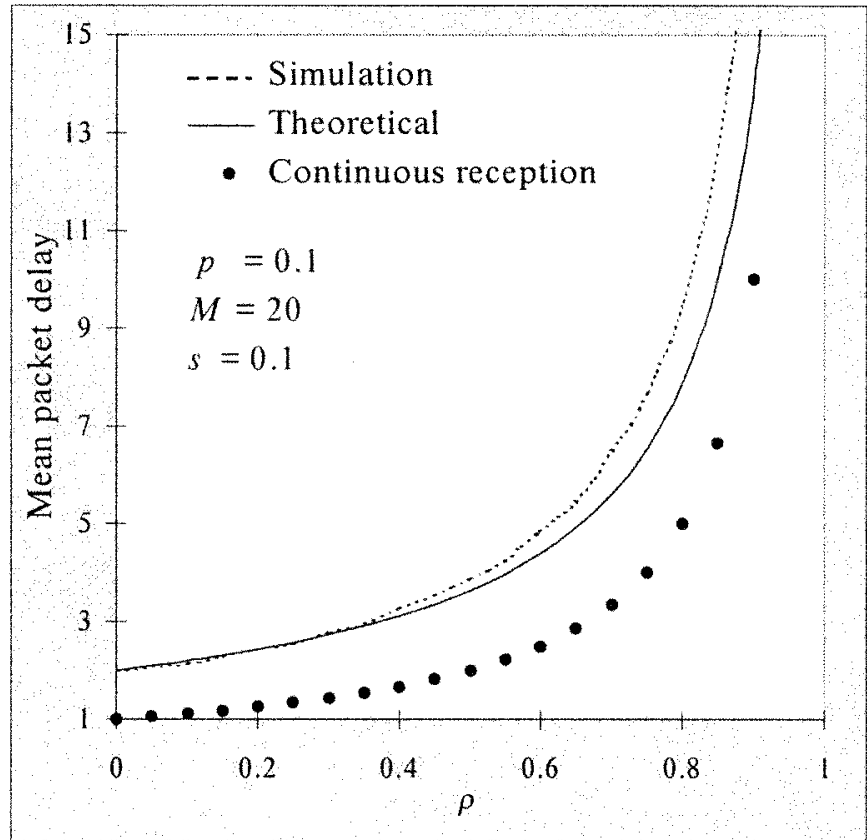

(b)

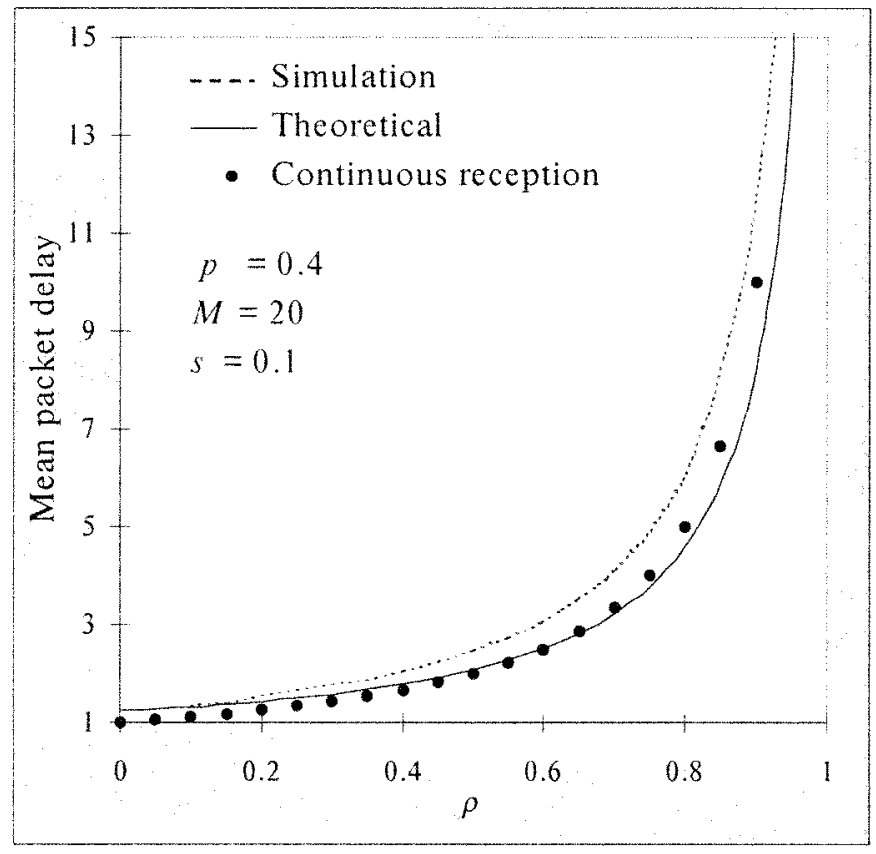

(d)

Fig. 5. Theoretical (solid lines) and simulation (dashed lines) results for the mean downlink packet delay in a network with $M=20$ terminals and $s=0.1$. The page reception probability varies from $p=0.05$ in (a) to $p=0.4$ in (d). The mean downlink packet delay of the continuous reception (•) is also displayed for comparison.

Before closing this section, we display below some other interesting theoretical results. Fig. 6 illustrates the probability the downlink channel to be in state $B$ (paging transmission) for a mobile network with $M=20$ terminals and $s=0.1$. Virtually, the curves shown represent the percentage of time where the downlink channel is used for paging. It is interesting to see that there is always a point after which this percentage of time is progressively reduced. The fact that the curves do not converge to zero when $\rho \rightarrow 1$ is due to the inaccuracies introduced by our simplifying assumptions.
Fig. 7 essentially displays (24) for several numbers of terminals $M$. It expresses the average number of terminals serviced from the time a terminal starts being paged until that terminal transmits an RRN message. It becomes evident that in the limiting case where $\rho \rightarrow 1$ a terminal being paged will send an RRN after all the rest terminals $(M-1)$ will have been serviced.

Finally, Figs. 8 and 9 depict the average number of packets transmitted per service period and the average paging delay $\bar{A}$ respectively. Both figures correspond to a mobile network with 


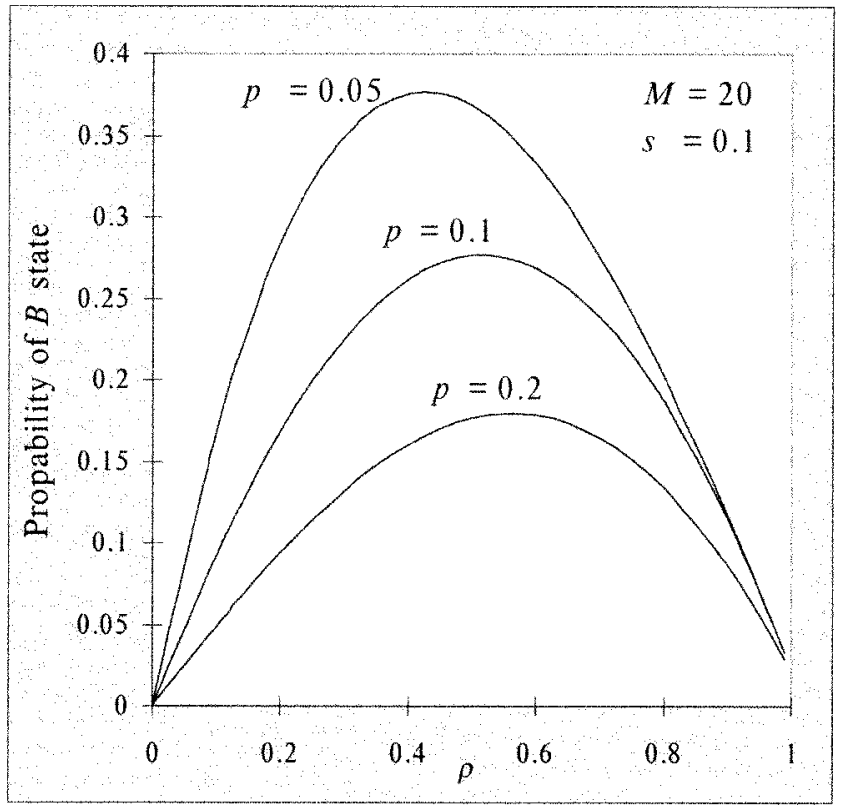

Fig. 6. Probability the downlink channel to be in the paging transmission state (state $B$ ) for a network with $M=20$ terminals and $s=0.1$. The various curves correspond to various page reception probabilities $p$.

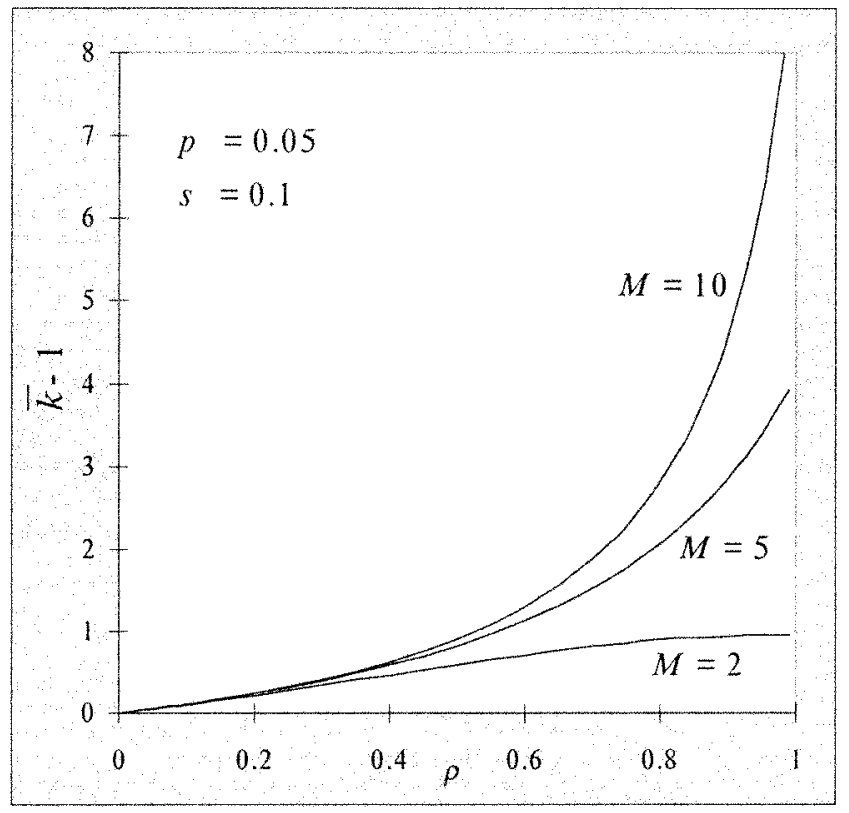

Fig. 7. Average number of terminals served during a paging delay period, i.e., from the time a terminal starts being paged until this terminal transmits an RRN message. Curves for $M=2,5$, and 10 terminals are displayed.

$M=20$ terminals and to page transmission time $s=0.1$. The various curves correspond to various reception probabilities $p$.

\section{B. Downlink Power Consumption}

Below we derive a simple closed expression of the average downlink power consumed by a terminal employing the IPS protocol. It must be pointed out that we only consider the downlink power consumption that is the power consumed for reception of data. The power consumed for transmission of data depends on

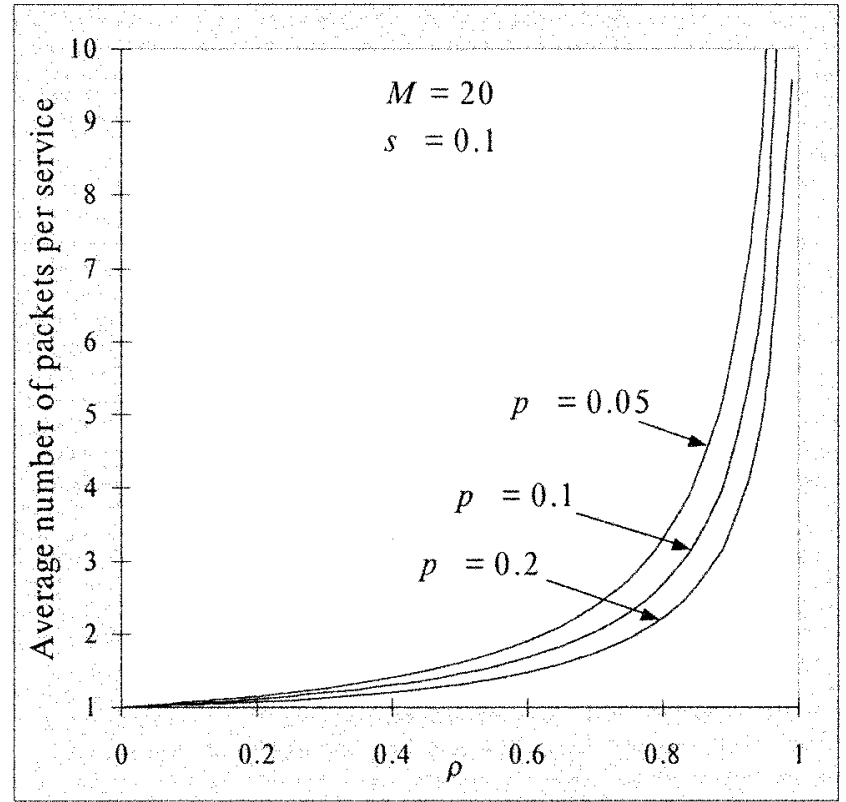

Fig. 8. Average number of transmitted packets per service period for three different page reception probabilities $(p=0.05,0.1$, and 0.2$)$ in a network with $M=20$ terminals and $s=0.1$.

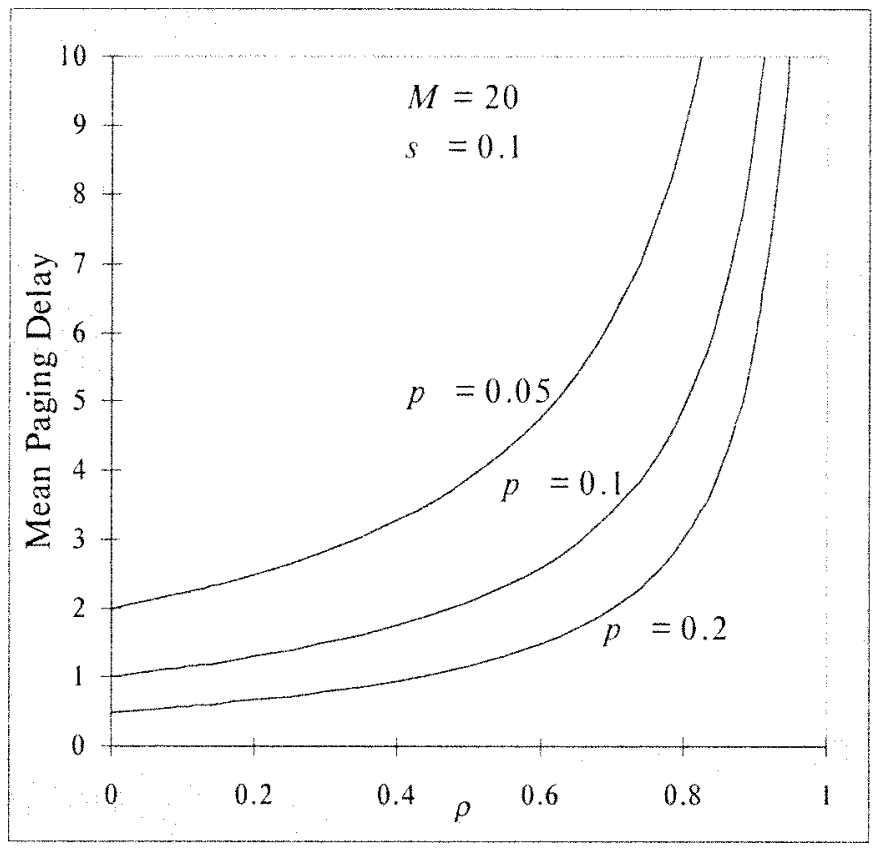

Fig. 9. Average paging delay $\bar{A}$ for three different page reception probabilities $(p=0.05,0.1$, and 0.2$)$ in a network with $M=20$ terminals and $s=0.1$.

the uplink MAC protocol and it is completely independent of the IPS protocol. ${ }^{6}$

As shown before, the probability the downlink channel to be in data transmission state (state $D$ ) is $\rho=\lambda / \mu$. Therefore, $\rho$ percentage of time the base station transmits data packets, and the rest of the time $(1-\rho)$ it transmits either page messages

${ }^{6} \mathrm{We}$ assume that when a terminal transmits a file to the base station, it stops the discontinuous operation of its receiver for as long as this file transmission takes. Also, the packets sent by the base station to acknowledge prior data transmissions are not transmitted through the IPS protocol but directly. 
or nothing. This means that, in the time unit, every terminal receives data for an average period of $\rho / M=\lambda_{s} / \mu$ while in the rest of time, $\left(1-\lambda_{s} / \mu\right)$, it is in power-saving mode operating discontinuously its receiver. According to (8), which expresses the relative occurrence frequency of state $D$, each terminal transits (again during the time unit) $\lambda_{s} /\left(\lambda_{s} \bar{A}+1\right)$ times to the data reception state, or equivalently, it transmits $\lambda_{s} /\left(\lambda_{s} \bar{A}+1\right) \mathrm{RRN}$ messages on the average.

From the above considerations we derive that the average downlink power consumption of a terminal can be expressed as

$$
\bar{P}=\left(\frac{\lambda_{s}}{\mu}\right) P_{r}+\left(1-\frac{\lambda_{s}}{\mu}\right) p P_{r}+\left(\frac{\lambda_{s}}{\lambda_{s} \bar{A}+1}\right) t_{\alpha} g P_{r}
$$

where $P_{r}$ is the power consumption of the receiver, $p$ is the page reception probability, and $t_{\alpha}$ is the duration of an RRN transmission and $g$ is the ratio $P_{t} / P_{r}$, where $P_{t}$ is the power consumption of the transmitter. ${ }^{7}$ At this point, we define the normalized downlink power consumption (NDPC) as the ratio of the average downlink power consumed by a terminal operating with the IPS protocol over the downlink power consumed by a terminal which continuously operates its receiver. That is

$$
\mathrm{NDPC}=\frac{\bar{P}}{P_{r}}=\left[\frac{1-p}{\mu}+\left(\frac{\lambda_{s}}{\lambda_{s} \bar{A}+1}\right) g t_{\alpha}\right] \lambda_{s}+p .
$$

In Fig. 10(a) and (b), the NDPC factor is displayed for a mobile network with 20 terminals operating with $p=0.1$ and with page transmission duration $s=0.1$. The RRN transmission time $t_{\alpha}$ is 0.5 in Fig. 10(a) and 0.1 in Fig. 10(b). The various curves shown correspond to various customary values of $g .{ }^{8}$ Also, in Fig. 11(a) and (b), the corresponding simulation results are depicted for comparison. It can be easily identified that the theoretical results exhibit the general downlink consumption behavior of the terminals however the are generally more pessimistic. This is because the theoretical model predicts a larger number of RRN transmissions per time unit.

As we note, the downlink power consumption of the terminals depends heavily on the average downlink traffic intensity. Initially, as this traffic increases the downlink consumption increases too, however (in most cases), there is a point after which the downlink consumption decreases due to the drastic reduction of RRN transmissions per time unit. Indeed, at very high traffic conditions the terminals will be virtually in continuous reception therefore when $\rho \rightarrow 1$ the curves corresponding to various values of $\mathrm{g}$ tend to converge to the same point.

In cases where the values of $t_{\alpha}$ and $g$ are quite large, it is possible for the factor NDPC to become larger than one which means that the protocol may exhibit larger downlink power consumption than a conventional continuous-reception system. However, this is not discouraging for two reasons. First, the combinations of $t_{\alpha}, g$, and $\rho$ values that may result to this effect are expected to be rare in practice. Second, this effect can be easily eliminated in practice when, for example, the terminals

\footnotetext{
${ }^{7}$ Parameter $g$ can also imbed any additional parameters that can affect the power consumption (e.g., the energy for powering up the antenna circuitry).

${ }^{8}$ As discussed in [7], the customary valued of $g$ range from 1 (for cordless telephones and common spread-spectrum transceivers) to 60 (for GSM phone and general-purpose VHF-UHF tranceivers).
}

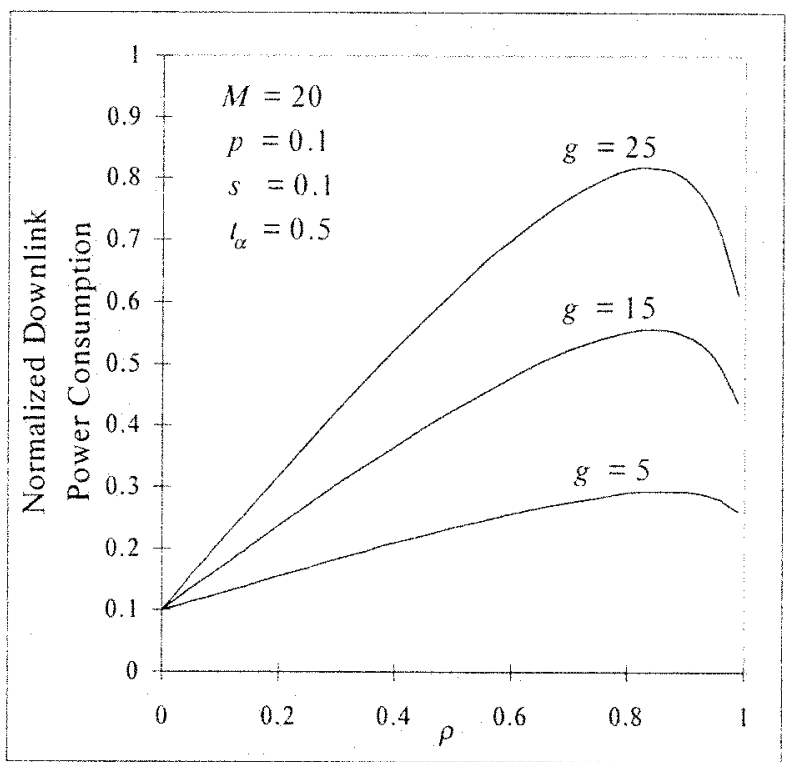

(a)

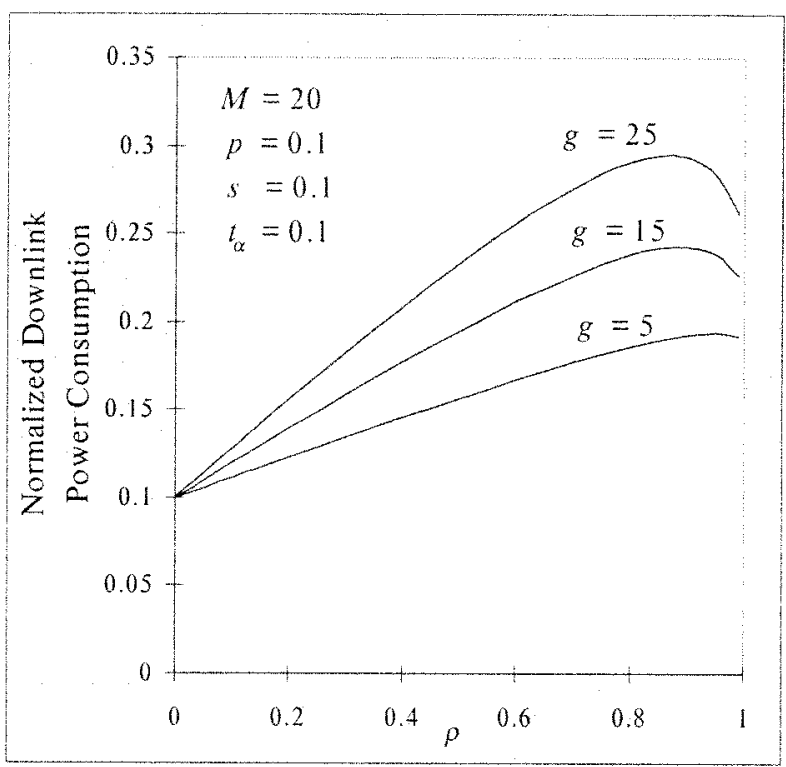

(b)

Fig. 10. Theoretical curves displaying the NDPC versus the normalized downlink traffic intensity $\rho$. Parameter $t_{\alpha}$ stands for the transmission duration of RRN messages, and $g$ stands for the transmission to reception power ratio. In (a) $t_{\alpha}=0.5$ and in (b) $t_{\alpha}=0.1$.

estimate the NDPC factor. When that estimation is found larger than one, terminals may change to continuous-reception mode.

Nevertheless, the important result is that the IPS protocol manifests considerable power-saving characteristics in the entire range of traffic conditions, and in practice it can provide beneficial power-saving properties.

\section{Nonfixed Page Reception Probability}

So far the page reception probability $p$ has been considered constant which means that the discontinuous operation of the terminals remained fixed and independent of the downlink traffic volume. Another interesting case, however, is when the 


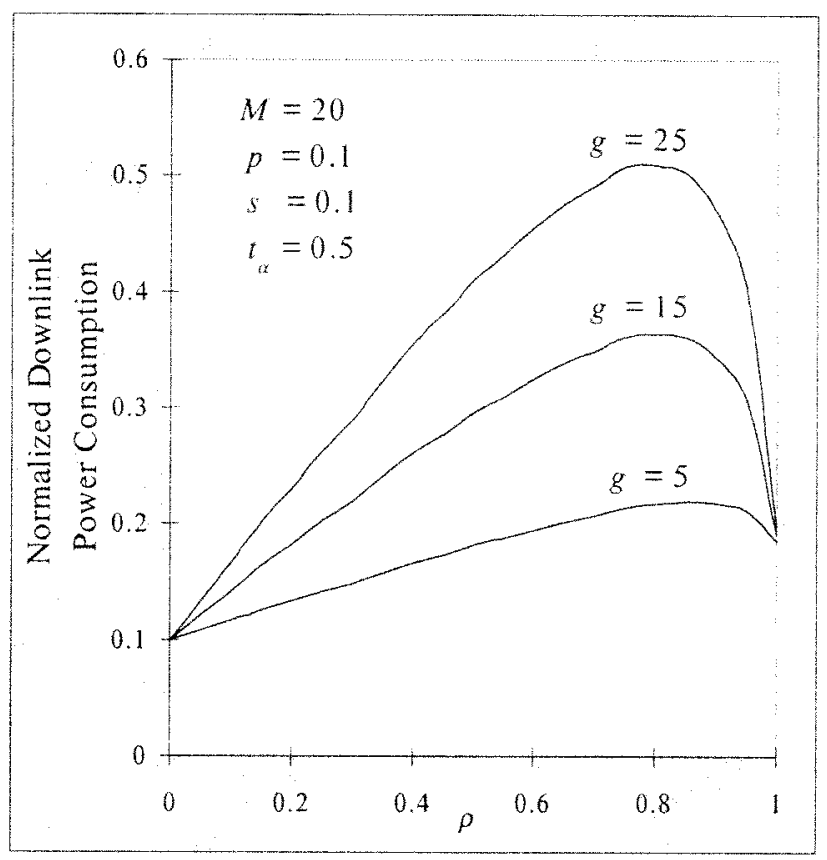

(a)

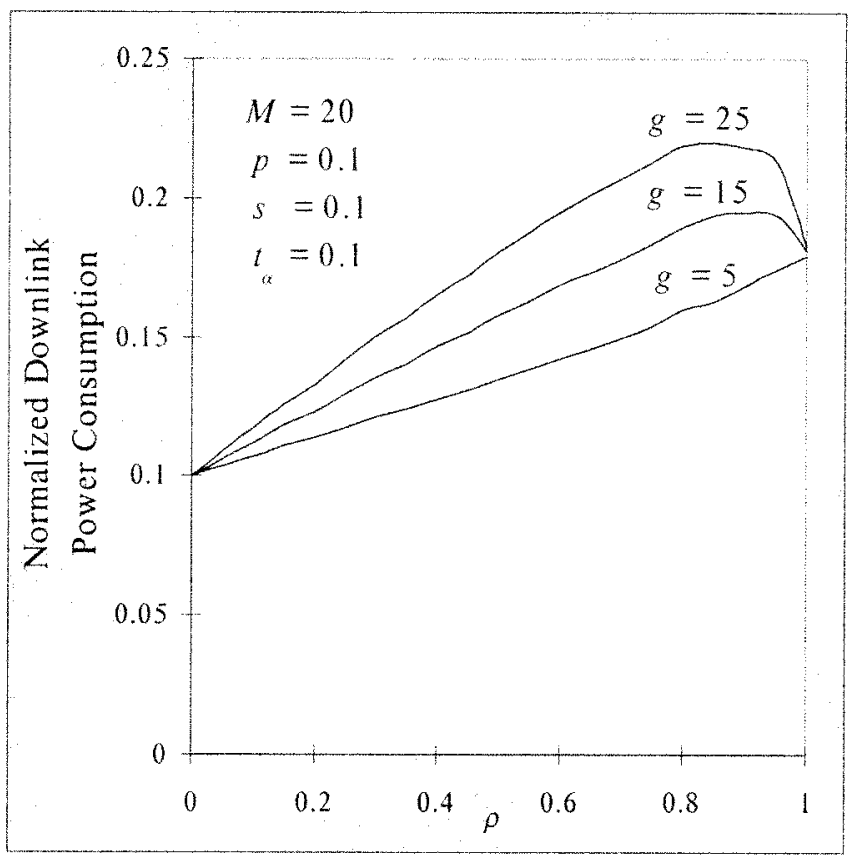

(b)

Fig. 11. Simulation curves displaying the NDPC versus the normalized downlink traffic intensity $\rho$. Parameters have the same meaning as in Fig. 10 , and the curves shown are for comparison to the corresponding curves of Fig. 10.

terminals adapt their discontinuous operation to the downlink traffic conditions. In such cases, the page reception probability $p$ would be small in light traffic conditions and it would approximate one in heavy traffic conditions. Assuming a linear dependence, the page reception probability can be modeled as

$$
p=p_{m}+\rho\left(1-p_{m}\right)
$$

where $p_{m}$ is the minimum value of $p$ used when $\rho=0$.

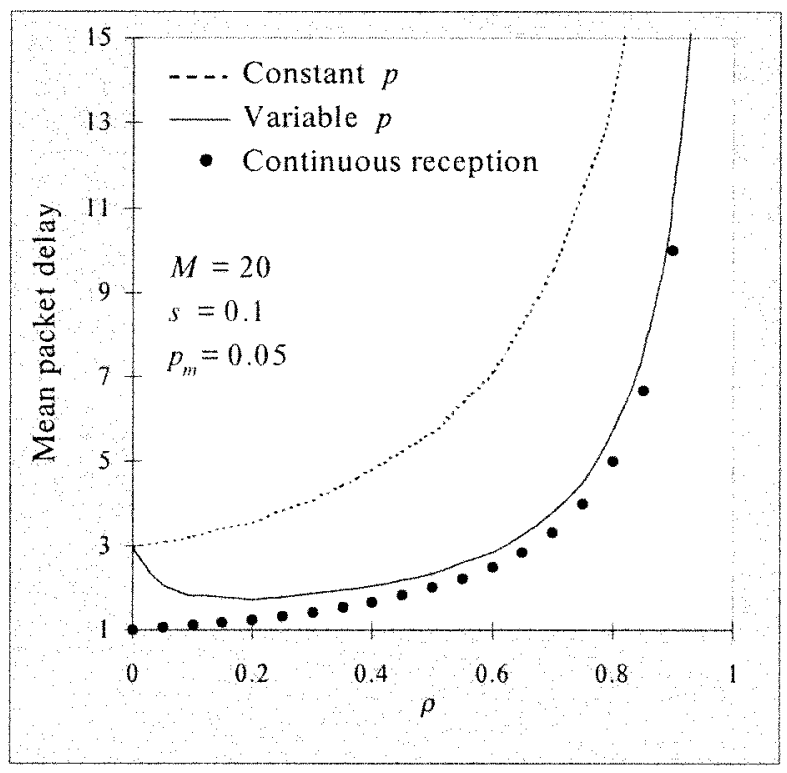

(a)

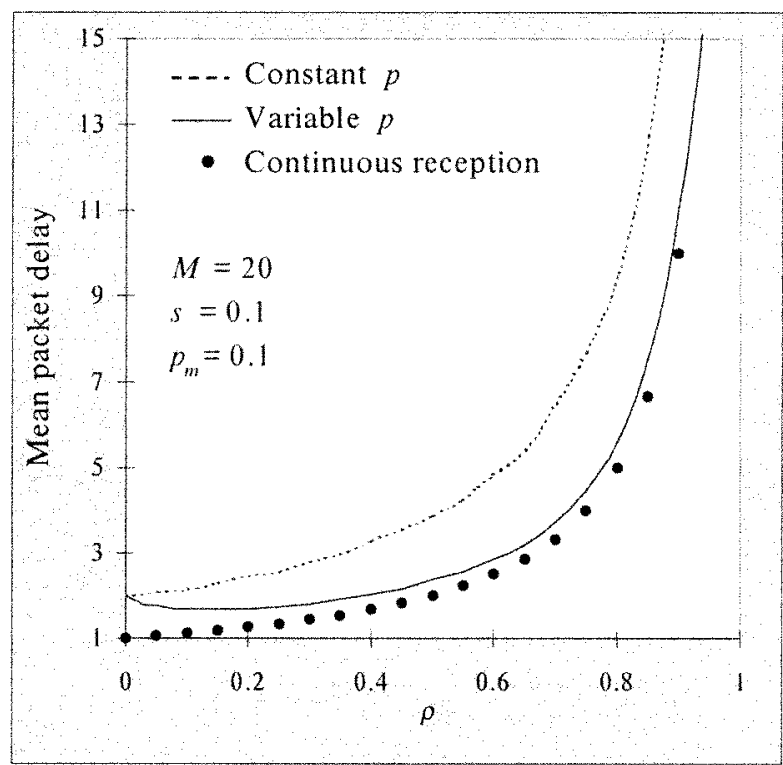

(b)

Fig. 12. Simulation results for the mean downlink packet delay for a network with $M=20$ terminals and $s=0.1$. Solid lines correspond to a system with variable page reception probability [according to (28)], and dashed lines correspond to the same system with fixed page reception probability $p=p_{m}$. In (a) $p_{m}=0.05$ and in (b) $p_{m}=0.05$. The mean downlink packet delay for the continuous reception $(\bullet)$ is also displayed for comparison.

Unfortunately, some of the approximations made in the above theoretical treatment of the IPS protocol cannot be used in this case. For example, the evaluation of $\bar{B}$ through the second-order polynomial form of (11) is not valid any more, and similar approximations proved to be far from acceptable. For this reason we resort to computer simulation to derive some performance parameters when (28) is in effect.

Fig. 12(a) and (b) illustrates the mean downlink packet delay versus the normalized downlink traffic $\rho$ for a system with $M=$ 20 terminals, with page transmission time $s=0.1$ and for 


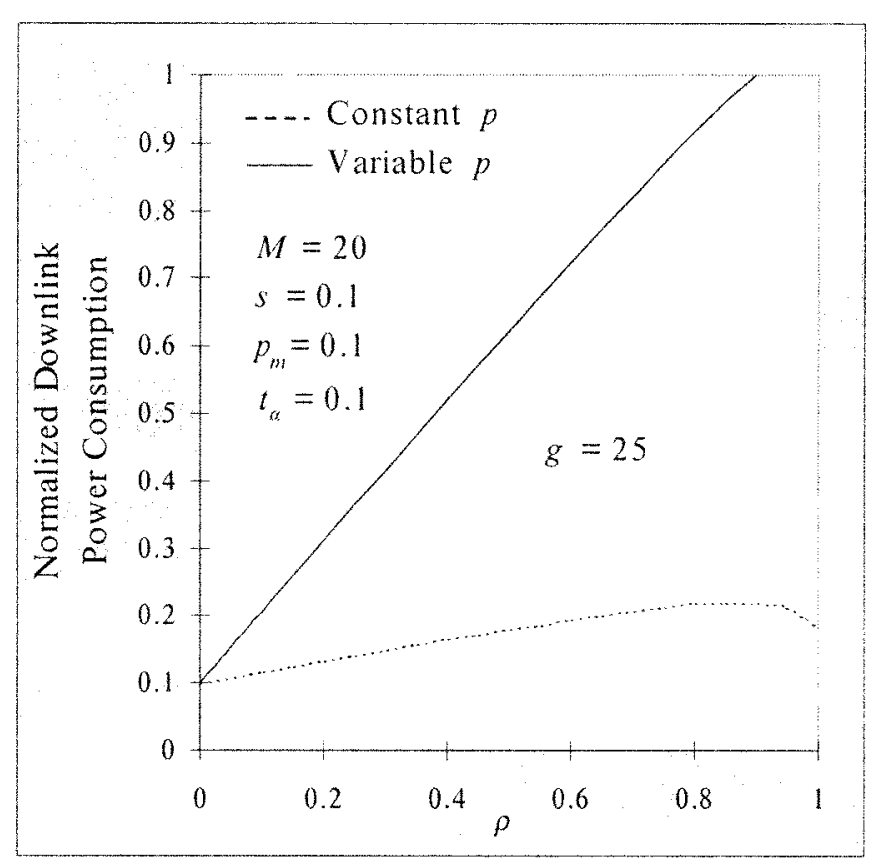

Fig. 13. Simulation curves displaying the NDPC versus the normalized downlink traffic intensity $\rho$ for a system with variable $p$ (solid line) and a system with constant $p$. Parameters have the same meaning as in Fig. 10.

$p_{m}=0.05$ and $p_{m}=0.1$, respectively. For comparison the mean packet delay is also illustrated for the continuous reception case and for the ISP with constant $p=p_{m}$. As expected, when the page reception probability is traffic dependent (variable) the mean packet delay is considerably smaller than when it is constant, and it quickly converges to the mean packet delay of the continuous reception. It is interesting to observe that at low traffic rates (typically $\rho<0.2$ ) the mean packet delay decreases as the downlink traffic increases. This is due to the dramatic reduction of the paging delay caused by the increase of the page reception probability according to (28). However, since the mean packet delay is the sum of a paging delay and a service delay, after a traffic point wherefore the service delay prevails the mean packet delay rapidly increases and approaches the continuous reception curve. Another interesting observation derived from Fig. 12(a) and (b) is that the minimum page reception probability $p_{m}$ affects the mean packet delay only in light traffic conditions. When the normalized downlink traffic gets larger than 0.2 , the mean packet delay gets almost independent of $p_{m}$.

Of course, the price we pay for the substantial reduction of the mean packet delay (as compared to the "constant $p$ " case) is a substantial increase of the downlink power consumption. This is clearly demonstrated in Fig. 13 where the NDPC factor is plotted against the normalized downlink traffic rate for the "constant $p$ " and the "variable $p$ " cases. When $p$ varies according to (28), the NDPC exhibits a linear dependence on the downlink traffic and increases rapidly until it reaches one. On the other hand, when $p$ is fixed and equal to $p_{m}$, the NDPC factor increases only slightly with the downlink traffic and remains well below the continuous reception case.

Typically, when the system shown in Fig. 13 transits from the constant $p$ case to the variable $p$ case, it will face a $215 \%$

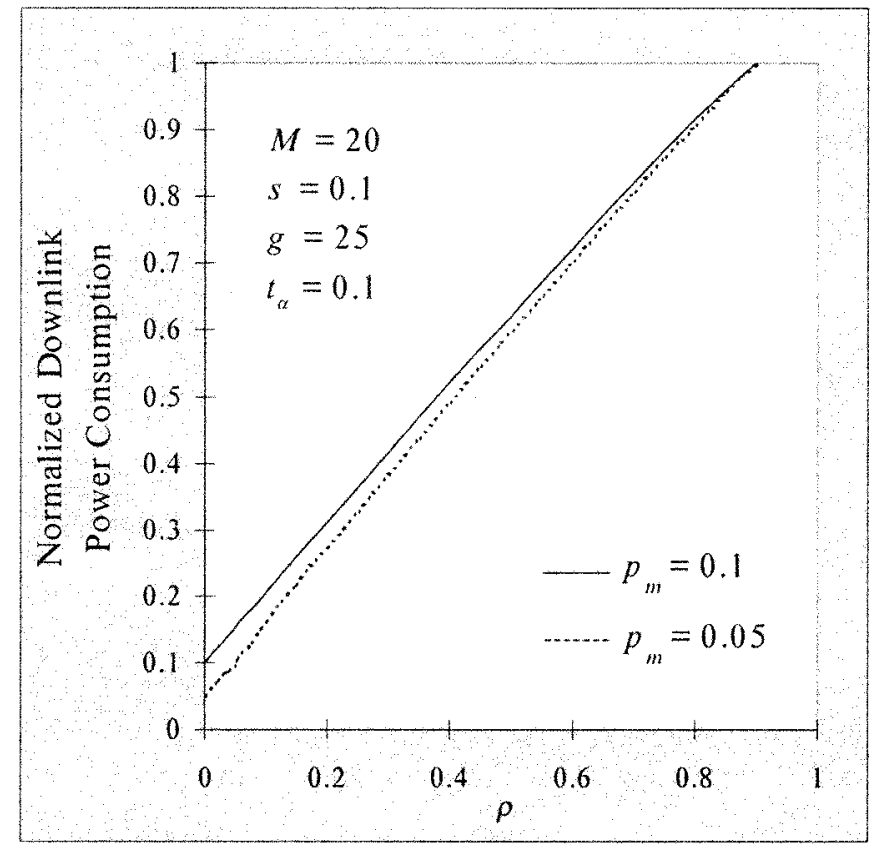

Fig. 14. Simulation curves displaying the NDPC versus the normalized downlink traffic intensity $\rho$ for a system with variable $p$ and for $p_{m}=0.1$ (solid line) and $p_{m}=0.05$ (dashed line).

increase of the NDPC and a $37 \%$ decrease of the mean packet delay at $\rho=0.4$. Except for the very low downlink traffic rates, the percentage increment of NDPC is far bigger than the corresponding percentage decrement of mean packet delay.

As with the mean packet delay, $p_{m}$ exhibits a minimum effect on the NDPC factor. This is clearly illustrated in Fig. 14 for $p_{m}=0.05$ and $p_{m}=0.1$. However, we observe that even for $\rho>0.2$ (where the mean packet delay gets practically independent of $p_{m}$ ), the NDPC is always smaller for $p_{m}=0.05$. Therefore, the choice of a small $p_{m}$ results in a beneficial effect for $\rho>0.2$; the downlink power consumption gets smaller (as compare to higher $p_{m}$ values) while the mean packet delay is approximately the same. For example, for the system shown in Fig. 14 and for $\rho=0.4$, the NDPC decreases by $5.8 \%$ when going from $p_{m}=0.1$ to $p_{m}=0.05$ while the mean packet delay increases by only $0.02 \%$.

\section{CONCLUSION}

This paper has mainly focused on the theoretical treatment of the IPS protocol, which is an asynchronous downlink MAC protocol that provided power-saving support features. Some important figures of merit of this protocol were analyzed and useful formulas for them were derived. More specifically, the mean downlink packet delay and the mean downlink power consumption of the protocol have been studied for two cases: when the discontinuous operation of the terminals is fixed and when it is traffic dependent. For the latter case, only simulation results were presented whereas for the former case both theoretical and simulation results were displayed and compared to each other.

Besides the theoretical analysis the main properties of the IPS protocol were demonstrated and discussed against the corresponding properties of similar but synchronous power-saving protocols. The main property that should be underlined again 
is the capability of the protocol to let wireless terminals regulate their own power consumption by monitoring the downlink channel whenever and as frequently as the want.

Regarding the derived theoretical results, it may be claimed that they follow quite closely the simulation results (specifically at low-to-medium downlink traffic rates), and therefore the presented analysis provides an acceptable theoretical tool for the IPS protocol.

As expected, the IPS protocol provokes increased packet delay on the downlink channel as a price for the attained power conservation. For example, with fixed page reception probability $p=0.1$ the mean packet delay is increased by two as compared to the continuous reception case, while with $p=0.2$ it is increased by 1.4. On the other hand, however, with $p=0.1$ the downlink power consumption of the terminals can be reduced by as much as $90 \%$ at low-traffic rates.

Finally, it turns out that the analyzed IPS protocol can provide an attractive alternative to the power-saving problem. It is easily implemented, and it could provide an asynchronous power-saving option to the wireless LAN's and to the mobile data networks.

\section{REFERENCES}

[1] M. Stemm et al., "Measuring and reducing energy consumption of network interfaces in hand-held devices," in Proc. MoMuC-3, Princeton, NJ, Sept. 1996.

[2] H. Woesner et al., "Power-saving mechanisms in emerging standards for wireless LANs: The MAC lever perspective," IEEE Personal Commun., vol. 5, pp. 40-48, June 1998.

[3] A. K. Salkintzis and C. Chamzas, "An in-band energy saving protocol for mobile data networks," IEEE Trans. Commun., vol. 46, pp. 1194-1205, Sept. 1998.

[4] MOBITEX Interface Specification, 1994.

[5] CDPD Specification 1.1, Jan. 1995.

[6] P802.11 Draft Standard for Wireless LAN: Medium Access Control (MAC) and Physical Layer (PHY) Specification, Apr. 1996.

[7] A. K. Salkintzis, "Efficient operation protocols for digital radio networks," Ph.D. dissertation, Democritus Univ. Thrace, Greece, Sept. 1997.

[8] A. K. Salkintzis, C. Chamzas, and C. Koukourlis, "An energy saving protocol for mobile data networks," presented at the 5th Int. Conf. Advances in Communication Control, COMCON 5, Rethymno, Greece, June 26-30, 1995.

[9] A. K. Salkintzis and C. Chamzas, "An outband paging protocol for energy-efficient mobile computing,", to be published.

[10] J. R. Lorch and A. J. Smith, "Software strategies for portable computer energy management," IEEE Personal Commun., vol. 5, pp. 60-73, June 1998.

[11] L. E. Larson, "Radio frequency integrated circuit technology for lowpower wireless communications," IEEE Personal Commun., vol. 5, pp. 11-19, June 1998.

[12] T. H. Meng et al., "Low-power signal processing system design for wireless applications," IEEE Personal Commun., vol. 5, pp. 20-31, June 1998.

[13] N. Bambos, "Toward power-sensitive network architectures in wireless communications: Concepts, issues, and design aspects," IEEE Personal Commun., vol. 5, pp. 50-59, June 1998.
[14] H. Kobayashi and A. G. Konheim, "Queueing models for computer communications system analysis," IEEE Trans. Commun., vol. COM-25, pp. 2-28, 1977.

[15] L. Kleinrock, Queueing Systems Vol. II: Computer Applications. New York: Wiley-Interscience, 1976.

[16] High Performance Radio Local Area Network (HIPERLAN), ETSI Draft standard ETS 300 652, Mar. 1996.

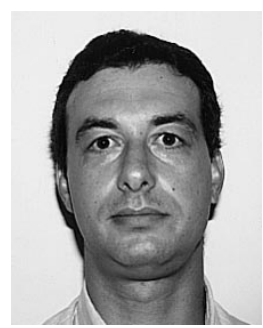

Apostolis K. Salkintzis (M'98) received the Diploma in 1991 and the Ph.D. degree in 1997, both from the Democritus University of Thrace, Xanthi, Greece.

From 1992 to 1997, he was a Research Engineer at Democritus University. In 1998, he joined the Department of Electrical and Computer Engineering, University of British Columbia, Vancouver, B.C., Canada, as a Post-Doctoral Fellow. Since January 1999, he has also been a Lecturer at the University of British Columbia. He is currently a Visiting Fellow of the British Columbia Advanced Systems Institute (ASI). His primary research interests lie in the area of wireless digital communication systems and networks. In particular, he is interested in mobile channel modeling, smart antennas and diversity techniques in wireless communication systems, error-correction coding, adaptive channel equalization, radio modem design with DSP's, mobility management, and multiple-access and data link protocols. He served as the Principal Guest Editor of the "Special Issue on the Evolution of Mobile Data Networking" for the IEEE Personal Communication MAGAZINE.

Dr. Salkintzis is a member of the Technical Chamber of Greece. He also served as the Principal Guest Editor of the "Special Issue on Mobile Data Networks: Advanced Technologies and Services" for the ACM/Baltzer Mobile Networks and Applications Journal.

Christodoulos Chamzas (SM'85) was born in Komotini, Greece. He received the Diploma degree in electrical and mechanical engineering from the National Technical University of Athens, Athens, Greece, in 1974 and the M.S. and Ph.D. degrees in electrical engineering in 1975 and 1979, respectively, from the Polytechnic Institute of New York, Farmingdale.

From 1979 to 1982, he was an Assistant Professor with the Department of Electrical Engineering, Polytechnic Institute of New York. In September 1982, he joined AT\&T Bell Laboratories, Holmdel, NJ, where he was a Member of the Visual Communications Research Department until 1990, where he worked on adaptive systems, mobile communications, multimedia image databases, and image coding. Since September 1990, he has been a Member of the Faculty of the Electrical and Computer Engineering Department, Democritus University of Thrace, Xanthi, Greece, where he is Director of the Electric Circuits Analysis Laboratory of the Image Processing and Multimedia Unit and Director of the Cultural and Educational Technologies Institute. He was instrumental in the definition, design, and implementation of the CCITT/ISO (JBIG, JPEG, etc.) standards for coding, storage, and retrieval of images (color and bilevel), an area where he holds six international patents. From 1985 to 1986, he was a Visiting Professor with the Department of Computer Science, University of Crete, Iraklion, Greece. Since 1994, he has been a Member of the Telematics for Knowledge Working Party of EE. He has held summer positions in Greece, England, and Portugal as well as at Bell Laboratories. His primary interests are in digital signal processing, image coding, multimedia, and communications systems. He is currently interested in the implementation of multimedia image database algorithms with either fast software or VLSI design. He was an Associate Editor for the IEEE TRANSACTIONS ON COMMUNICATIONS.

Dr. Chamzas is a Member of the Technical Chamber of Greece and Sigma Xi. 